\title{
Síndrome neuroléptico maligno debido ziprasidona: reporte de un caso
}

\author{
Neuroleptic malignant syndrome due to ziprasidone: a case report \\ Jeff Huarcaya-Victoria ${ }^{1,2,3, a, b}$, Claudia Castillo ${ }^{2, c, d}$, Dwight Herrera ${ }^{2, c, e}$
}

\section{RESUMEN}

El síndrome neuroléptico maligno es una rara y potencialmente fatal reacción adversa medicamentosa, asociada usualmente al uso de antipsicóticos. Tiene por características la presencia de fiebre, rigidez muscular, estado mental alterado y disfunción autonómica. Los hallazgos de laboratorio son inespecíficos, sin embargo, la presencia de leucocitosis y elevación de la creatina fosfoquinasason hallazgos frecuentes. Presentamos el caso de un paciente varón de 51 años, natural de Lima con antecedentes médicos de tuberculosis pulmonar y esquizofrenia que acude a nuestro hospital con un cuadro de psicosis quien, luego de ser tratado con ziprasidona administrada por vía intramuscular, presentó los síntomas característicos de un síndrome neuroléptico maligno. Conocer la clínica y la fisiopatología de este síndrome nos permitirá un mejor abordaje, ya que por su poca frecuencia podría no llegar a plantearse dentro de los diagnósticos diferenciales, lo que resultaría perjudicial para el paciente.

PALABRAS CLAVE: Síndrome neuroléptico maligno, ziprasidona, psicosis, esquizofrenia.

\section{SUMMARY}

Neuroleptic malignant syndrome is a rare and potentially fatal drug adverse reaction, usually associated with antipsychotics. Signs and symptoms include: fever, muscle rigidity, altered mental status and autonomic dysfunction. Laboratory findings are nonspecific, however the presence of leukocytosis and elevated creatinine phosphokinase are frequent findings. We present the case of a 51-year-old male patient from Lima with a medical history of pulmonary tuberculosis and schizophrenia that comes to our hospital with psychotic symptoms. After being treated with ziprasidone, administered by intramuscular injection presents with typical symptoms of neuroleptic malignant syndrome. Knowing the clinical features and the pathophysiology of this syndrome will allow us to better approach the condition. Due to its infrequent presentation, it may not be considered within the differential diagnosis, which could be harmful to the patient.

KEYWORDS: Neuroleptic malignant syndrome, ziprasidone, psychosis, schizophrenia.

1 Universidad de San Martín de Porres, Facultad de Medicina, Centro de Investigación en Salud Pública. Lima, Perú.

2 Departamento Académico de Psiquiatría, Facultad de Medicina de San Fernando, Universidad Nacional Mayor de San Marcos. Lima, Perú.

3 Departamento de Psiquiatría, Hospital Nacional Guillermo Almenara Irigoyen, EsSalud. Lima, Perú.

a Médico psiquiatra, Coordinador de la Unidad de Psiquiatría de Enlace.

b ORCID: https://orcid.org/0000-0003-4525-9545

c Médico residente de psiquiatría.

d ORCID: https://orcid.org/0000-0002-6277-7152

e ORCID: https://orcid.org/0000-0002-6456-8717 


\section{INTRODUCCIÓN}

El síndrome neuroléptico maligno (SNM) es una rara y potencialmente fatal reacción adversa medicamentosa, comúnmente asociada a medicamentos antipsicóticos (neurolépticos)(1). Se caracteriza por la presencia de fiebre, rigidez muscular, estado mental alterado y disfunción autonómica. Los hallazgos de laboratorio son inespecíficos, no obstante, la leucocitosis y elevación de la creatina fosfoquinasa (CPK) son frecuentes. El SNM es impredecible, no tiene marcadores clínicos establecidos y, a menudo, se presenta como un trastorno emergente que amenaza la vida del paciente, por lo que es dificultoso obtener un consentimiento informado para participar en protocolos de investigación. Estas características explican los pocos estudios clínicos en esta entidad, siendo su principal fuente de información los reportes o series de casos (2).

La incidencia reportada del SNM varió durante las últimas décadas, no obstante, siempre se caracterizó por su poca frecuencia. En 1993 se describió una incidencia alrededor del 0,2 al $3 \%$ (3), pero en los últimos años se ha observado una disminución, llegando hasta el 0,01 al $0,02 \%$ (4), posiblemente debido a un mayor cuidado en la prescripción y titulación de la medicación y al mayor uso de antipsicóticos atípicos. En un reciente metanálisis se encontró que el SNM se diagnostica con más frecuencia en la adultez temprana, y la frecuencia del diagnóstico disminuye gradualmente a lo largo del resto de la vida. Los varones tienen aproximadamente un $50 \%$ más de probabilidades de ser diagnosticados a cualquier edad (5). Esto podría atribuirse al mayor uso de antipsicóticos en los varones, la adherencia al tratamiento, diferencias farmacocinéticas y farmacodinámicas (6).

Inicialmente la etiología del SNM se relacionó con los neurolépticos (antipsicóticos de primera generación), es decir, aquellos psicofármacos que ayudaban a controlar los síntomas psicóticos y producían como efecto secundario síntomas extrapiramidales (7). De esta manera, se describió al SNM como un conjunto de signos y síntomas que se presentaban como una reacción adversa idiosincrática, potencialmente severa, secundaria al empleo de antipsicóticos de primera generación. No obstante, en el tiempo se ha visto que se puede presentar también debido al uso de antipsicóticos de segunda generación (atípicos), estabilizadores del humor y hasta medicación distinta a los psicofármacos, pudiendo presentar características atípicas, es decir, mostrar una menor intensidad en los síntomas extrapiramidales o fiebre intensa (8). En una revisión sistemática se encontró una menor incidencia de SNM producida por antipsicóticos de segunda generación, además de menor gravedad clínica y resultados letales poco frecuentes comparado con el uso de antipsicóticos de primera generación(8).

Con la finalidad de revisar brevemente la literatura sobre el SNM, presentamos el caso de un hombre de 51 años con antecedente de esquizofrenia que presentó un SNM luego de la administración de ziprasidona por vía intramuscular.

\section{Reporte de caso}

Se trata de un paciente varón de 51 años, natural de Lima. Soltero, con grado de instrucción secundaria completa, actualmente laborando como mozo en un restaurante. Es el mayor de una fratría de 4. Tiene como antecedentes médicos un diagnóstico de tuberculosis pulmonar multidrogo resistente desde el año 2003 hasta el año 2007, periodo en el cual presentó 3 recaídas; no se registró como antecedente el desarrollo de una meningoencefalitis tuberculosa, actualmente ya no recibe tratamiento antituberculoso.

Como antecedentes psiquiátricos se destaca un diagnóstico de esquizofrenia paranoide desde los 17 años, por lo que requiriómúltiples hospitalizaciones en el Hospital Nacional Guillermo Almenara Irigoyen, la última fue en marzo del 2016. Tiene una mala adherencia al tratamiento, con múltiples esquemas terapéuticos. Además, presenta un antecedente de síndrome extrapiramidal en forma de parkinsonismo. Su medicación habitual consiste en olanzapina $10 \mathrm{mg} /$ día, aripiprazol 7,5 mg/día, biperideno $4 \mathrm{mg} /$ día y propranolol $80 \mathrm{mg} /$ día.

Quince días antes del ingreso al servicio de emergencia de nuestro hospital el paciente refirió que lo "perseguían constantemente", además de escuchar una voz intimidante. Rehusaba asearse y no quería salir de su domicilio. Por todo esto es llevado al servicio de emergencia por sus familiares.

Al examen mental de ingreso encontramos a un paciente despierto, sin alteración del nivel de conciencia, que no establece contacto visual, orientado en las 3 esferas, con delusiones de daño, autorreferencial, ansioso, con sentimientos de frustración, hipobúlico, insomnio global y mal control de impulsos. Se decide su hospitalización 
Tabla 1. Niveles de CPK, leucocitos y constantes vitales durante la hospitalización del paciente.

\begin{tabular}{ccccccc}
\hline $\begin{array}{c}\text { Día de } \\
\text { hospitalización }\end{array}$ & $\begin{array}{c}\text { CPK } \\
\text { U/L }\end{array}$ & Leucocitos & $\begin{array}{c}\text { Presión } \\
\text { arterial }\end{array}$ & $\begin{array}{c}\text { Frecuencia } \\
\text { cardiaca }\end{array}$ & $\begin{array}{c}\text { Frecuencia } \\
\text { respiratoria }\end{array}$ & Temperatura \\
\hline 1 & --- & --- & $110 / 60$ & 74 & 16 & 36,6 \\
2 & 53 & 10000 & $150 / 90$ & 102 & 23 & 36,7 \\
3 & 7536 & 12980 & $140 / 80$ & 140 & 19 & 38 \\
8 & 5002 & --- & --- & --- & --- & --- \\
15 & 101 & 8810 & $120 / 80$ & 100 & 22 & 36,5 \\
18 & 566 & 14380 & $140 / 78$ & 108 & 22 & 38 \\
19 & 1255 & --- & $180 / 100$ & 140 & 26 & 37,7 \\
20 & 1127 & 7670 & $200 / 90$ & 148 & 24 & 37,8 \\
22 & 729 & 10000 & $161 / 90$ & 88 & 20 & 37,5 \\
24 & 889 & 10310 & $168 / 94$ & 99 & 22 & 38,2 \\
30 & 75 & 10880 & $167 / 86$ & 124 & 26 & 37,6 \\
33 & 80 & --- & $136 / 71$ & 80 & 20 & 38,2 \\
36 & 130 & 15330 & $162 / 90$ & 96 & 24 & 38,5 \\
37 & 692 & --- & $100 / 70$ & 112 & 22 & 37,2 \\
39 & 178 & 12640 & $150 / 70$ & 104 & 22 & 38,3 \\
40 & 160 & --- & $170 / 90$ & 104 & 28 & 37,4 \\
45 & 46 & 6010 & $130 / 80$ & 86 & 18 & 37,4 \\
50 & 17 & 7790 & $110 / 60$ & 109 & 18 & 37,4 \\
52 & 70 & 8440 & $120 / 80$ & 110 & 20 & 37 \\
54 & 17 & --- & $110 / 70$ & 110 & 19 & 36,9 \\
57 & 15 & 7230 & $130 / 80$ & 100 & 22 & 36,6 \\
63 & 19 & 5820 & $120 / 80$ & 104 & 21 & 37 \\
64 & 16 & 5450 & $120 / 80$ & 100 & 20 & 23 \\
70 & 17 & 5790 & $130 / 90$ & 99 & & 36,9 \\
\hline & & & & & 22 & \\
\hline
\end{tabular}

en emergencia, con las siguientes indicaciones: ziprasidona $20 \mathrm{mg} 1$ ampolla intramuscular c/12 h y diazepam vía oral $20 \mathrm{mg}$ /día.

Luego de 24 horas de instaurado el tratamiento, el paciente presentó una elevación de la presión arterial a un valor de 150/90 $\mathrm{mmHg}$, así como una elevación de la frecuencia cardiaca a 102 latidos/min y diaforesis. A las 48 horas de iniciado el tratamiento con ziprasidona se agregó fiebre $\left(38^{\circ} \mathrm{C}\right)$. Se le instauró un tratamiento antihipertensivo, a pesar de lo cual persistióel cuadro hipertensivo.

Ante la sospecha de un SNM, se decidió suspender el antipsicótico y se solicitaron los exámenes de laboratorio correspondientes.Se encontró un valor de CPKde $7536 \mathrm{U} / \mathrm{L}$, y 12980 leucocitos. En los días posteriores persistieron los síntomas disautonómicos, además se agregaron síntomas catatónicos: negativismo, ecolalia, excitación catatónica, rigidez muscular, almohada psicológica y ecofenómenos. Se realizó la Bush-Francis Catatonia Rating Scale (BFCRS), puntuándose en 30. En la tabla 1 encontramos la evolución del CPK, leucocitos y constantes vitales. No se evidenció una disminución del flujo urinario ni falla renal.

A los 18 días de iniciado el cuadro, el paciente fue ingresado a la unidad de cuidados intensivos (UCI) de nuestro hospital con tubo orotraqueal más ventilación mecánica por un cuadro de insuficiencia respiratoria debido a una neumonía aspirativa intrahospitalaria y crisis hipertensiva. En la UCI se instauró el tratamiento con bromocriptina a una dosis de $1.25 \mathrm{mg}$ cada 8 horas por sonda nasogástrica (SNG), clonazepam 10 $\mathrm{mg} /$ día por SNG, así mismo se le indicó ceftazidima 
y ciprofloxacino endovenoso por la neumonía. En los días posteriores se aumentó la dosis de bromocriptina llegando hasta $30 \mathrm{mg} /$ día, se amplió la cobertura antibiótica a meropenen endovenoso y se aumentó la dosis de clonazepamhasta $12 \mathrm{mg}$ al día por SNG.

A los 45 días de hospitalización el paciente presentó una evolución favorable, se le retiró la ventilación mecánica, la rigidez desapareció, comenzó a presentar un habla espontánea. Se le retiró la bromocriptina, cumplió con su tratamiento antibiótico y se redujo la dosis de clonazepam a $6 \mathrm{mg} / \mathrm{dí}$, con lo cual fue dado de alta de UCI y transferido al servicio de psiquiatría de nuestro hospital; en donde se reevaluaron los síntomas catatónicos mediante la BFCRS, puntuando 11. Se decidió instaurar Olanzapina a dosis bajas, la cual se fue incrementando progresivamente, llegando a 20 $\mathrm{mg} /$ día. El paciente requirió permanecer hospitalizado por 30 días adicionales, debido a la persistencia de los síntomas psicóticos. Actualmente el paciente sigue sus controles en la consulta externa de psiquiatría de nuestro hospital, evidenciándose una buena evolución respecto a sus síntomas psicóticos y catatónicos.

\section{DISCUSIÓN}

Presentamos el caso de un paciente varón con manifestaciones típicas del SNM. El paciente cumplió los criterios diagnósticos del DSM-5 para un SNM asociado al uso de ziprasidona.

La ziprasidona, al igual que los demás antipsicóticos de segunda generación, tiene un perfil farmacológico complejo. Posee un cociente 5- $\mathrm{HT}_{2} /$ $\mathrm{D}_{2}$ elevado, una acción agonista potente del receptor $5-\mathrm{HT}_{1 \mathrm{~A}}$, antagonista de los receptores $5-\mathrm{HT}_{2 \mathrm{C}}$ y $5-\mathrm{HT}_{1 \mathrm{D}}$ y un moderado efecto inhibidor de la recaptación de serotonina y noradrenalina(9).

El mecanismo subyacente a los síntomas observados en el SNM probablemente esté relacionado con un bloqueo excesivo de los receptores dopaminérgicos, principalmente el D2. La antagonización de este receptor en las vías nigroestriatal, hipotalámica y mesolímbica/cortical probablemente explicaría respectivamente la rigidez, la hipertermia y la alteración del estado mental (1). Sin embargo, la idea de que el SNM se deba únicamente al bloqueo excesivo del receptor D2 puede resultar demasiado simplista, ya que no tiene en cuenta la rara aparición del síndrome y su inicio impredecible, incluso en pacientes con episodios previos (10). Otros cofactores pueden incluir el desequilibrio de los sistemas de norepinefrina, GABA, y serotonina, lo cual explicaría su aparición en antipsicóticos de segunda generación como la ziprasidona(10). La hiperactividad central simpato-suprarrenal también se ha postulado como una explicación probable de la etiología del SNM. El estado hipermetabólico es posiblemente el resultado de un exceso de noradrenalina en relación con la dopamina. Además, estudios recientes en farmacogenética indican un posible papel para la predisposición genética del SNM, por lo que es necesario obtener una adecuada historia familiar (11).

Las características clínicas podrían variar dependiendo del antipsicótico causante del SNM. Se ha descrito que la rigidez, tremor y fiebre son menos frecuentes con los antipsicóticos atípicos, mientras que la diaforesis es relativamente común. La clozapina, aripiprazol y amisulprida estarían asociadas particularmente con presentaciones atípicas del SNM, con infrecuentes elevaciones del CPK (8, $12,13)$. La risperidona produce un cuadro clínico similar al SNM producido por antipsicóticos típicos. Algunos antipsicóticos atípicos han sido asociados con características graves como la mioglobinuria o falla renal aguda (13).

En una revisión sistemática se encontraron 6 casos reportados de SNM por ziprasidona en la literatura. La edad media de los pacientes fue de 35 años, en su mayoría mujeres. La aparición del SNM fue generalmente abrupto, presentándose con síntomas típicos: alteración del estado mental, diaforesis, leucocitosis, tremor, incremento del CPK; sin embargo, solo en la mitad de los pacientes se encontró una temperatura mayor a $38^{\circ} \mathrm{C}$. En algunos casos la rigidez no estuvo presente. La evolución de estos pacientes fue favorable, mejorando dentro de los 10 primeros días (8).

En nuestro paciente fueron llamativos los síntomas catatónicos: estupor, mutismo, negativismo, flexibilidad cérea, verbigeración, ecofenómenos. Se ha documentado una fuerte correlación entre la severidad del SNM y la presencia de síntomas catatónicos (14). Para algunos investigadores, la forma letal de la catatonía es clínicamente indiferenciable del SNM (15). Para otros, la catatonía sería un síndrome psicomotor cortical, mientras que el SNM sería un síndrome motor subcortical (16).

Respecto al tratamiento, la evidencia científica es limitada ya que los fármacos empleados han sido reportados solo en casos individuales o pequeñas series 
de casos, además de la alta morbilidad y mortalidad en el SNM (6). Se deben garantizar medidas de soporte básicas y monitorio constante de las funciones vitales en unidades de cuidados intensivos, además se debe suspender inmediatamente el fármaco sospechoso de causar el SNM. Las benzodiacepinaspueden ser útiles para el alivio de la agitación o síntomas catatónicos (6). La bromocriptina estimula los receptores dopaminérgicos tipo 1 y antagoniza los receptores tipo 2 en el hipotálamo y la vía nigroestriada del sistema nervioso central. Puede utilizarse $2.5-5 \mathrm{mg}$ de forma oral o por sonda nasogástrica cada 8 horas. Puede incrementarse hasta $30-45 \mathrm{mg} /$ día de ser necesario (17). La suspensión prematura de la bromocriptina ha dado lugar a síntomas de rebote en algunos casos (18). El dantroleno es un relajante muscular utilizado en el tratamiento de la hipertermia maligna. Se puede utilizar de 1 a 2,5 mg / $\mathrm{kg}$ inicialmente por infusión intravenosa, seguido de infusión de $1 \mathrm{mg} / \mathrm{kg}$ cada 6 horas (máximo $10 \mathrm{mg} / \mathrm{kg} /$ día). En una revisión sistemática de reportes de caso se encontró que la monoterapia con dantroleno ha sido reportada como efectiva hasta en el $76,7 \%$ de los casos dentro de las primeras 24 horas, sin embargo, también ha sido reportado una mayor mortalidad respecto a otros tratamientos (16,2\%) (19). La ECT Se ha utilizado para tratar el SNM grave, o aquellos casos en los que el diagnóstico psiquiátrico subyacente es la depresión psicótica o la catatonía, y en los casos en los cuales no se pueda descartar una catatonía letal (20). Puede ser considerada como un tratamiento de segunda línea, administrándose si las medidas de soporte y tratamiento farmacológico falla durante los primeros siete días. En un estudio realizado a 48 pacientes con SNM se encontró que la ECT ha sido asociada con una disminución no significativa de la mortalidad en comparación con aquellos que recibieron un tratamiento psicofarmacológico (21). En una revisión de 55 reportes de caso se encontró que el tratamiento con 10 sesiones de ECT produjo una recuperación completa o parcial en el $90 \%$ de los pacientes (20).

En suma, la presentación del SNM, si bien es poco frecuente, resulta potencialmente fatal, incluso cuando se prescriben los antipsicóticos atípicos nuevos y "más seguros". El inicio de un SNM puede ser rápido, incluso luego de una dosis única, y el curso puede extenderse por varios meses. Una vez se sospeche de un SNM, se debe suspender inmediatamente el posible fármaco causante, se deben garantizar medidas de soporte adecuadas y administrar benzodiacepinas, además de considerar terapias complementarias como la bromocriptina y el dantroleno. El tratamiento de estos pacientes debe estar a cargo de un equipo multidisciplinario en una UCI, que garantice la efectiva monitorización del paciente.

Consideraciones éticas: Los familiares del paciente descrito brindaron su consentimiento para la publicación de su historial clínico.

Contribuciones de autorías: JHV, CC, participaron en la concepción del artículo; JHV realizó el diseño y redacción del artículo; $\mathrm{CC}, \mathrm{DH}$ facilitaron el resumen de la historia clínica. JHV, CC, DH realizaron la revisión crítica del artículo y aprobación de la versión final.

Fuentes de financiamiento: Autofinanciado.

Conflicto de intereses: Los autores declaran no tener conflicto de interés.

\section{Correspondencia:}

Jeff David Huarcaya Victoria

Universidad de San Martín de Porres - Instituto de Investigación

Av. Alameda del Corregidor, 1531, La Molina, Lima

Teléfono: 511-3653640

Correo electrónico: jhuarcayav@usmp.pe

\section{REFERENCIAS BIBLIOGRÁFICAS}

1. Pileggi DJ, Cook AM. Neuroleptic Malignant Syndrome. Ann Pharmacother. 2016;50(11):973-81. doi:10.1177/1060028016657553

2. Gurrera RJ, Simpson JC, Tsuang MT. Metaanalytic evidence of systematic bias in estimates of neuroleptic malignant syndrome incidence. Compr Psychiatry. 2007;48(2):205-11. doi:10.1016/j. comppsych.2006.10.004

3. Vurucu S, Congologlu A, Altun D, Unay B, Akin R. Neuroleptic malignant syndrome due to risperidone treatment in a child with Joubert syndrome. J Natl Med Assoc. 2009;101(3):273-5.

4. Berman BD. Neuroleptic malignant syndrome: a review for neurohospitalists. Neurohospitalist. 2011;1(1):41-7. doi:10.1177/1941875210386491

5. Gurrera RJ. A systematic review of sex and age factors in neuroleptic malignant syndrome diagnosis frequency. Acta Psychiatr Scand. 2017;135(5):398408. doi:10.1111/acps.12694

6. Oruch R, Pryme IF, Engelsen BA, Lund A. Neuroleptic malignant syndrome: an easily overlooked neurologic emergency. Neuropsychiatric disease and treatment. 2017;13:161-75. doi:10.2147/ NDT.S118438

7. Silva RR, Munoz DM, Alpert M, Perlmutter IR, 
Diaz J. Neuroleptic malignant syndrome in children and adolescents. J Am Acad Child Adolesc Psychiatry. 1999;38(2):187-94. doi:10.1097/00004583-199902 000-00018

8. Belvederi Murri M, Guaglianone A, Bugliani M, Calcagno P, Respino M, Serafini G, et al. Secondgeneration antipsychotics and neuroleptic malignant syndrome: systematic review and case report analysis. Drugs R D. 2015;15(1):45-62. doi:10.1007/s40268014-0078-0

9. Trollor JN, Chen X, Sachdev PS. Neuroleptic malignant syndrome associated with atypical antipsychotic drugs. CNS Drugs. 2009;23(6):477-92. doi:10.2165/00023210-200923060-00003

10. Mann S, Caroff S, Fricchione G, Campbell E. Central Dopamine Hypoactivity and the Pathogenesis of Neuroleptic Malignant Syndrome. Psychiatr Ann. 2000;30:363-74. doi:10.3928/0048-5713-2000050114

11. Velamoor R. Neuroleptic malignant syndrome: A neuro-psychiatric emergency: Recognition, prevention, and management. Asian J Psychiatr. 2017;29:106-9. doi:10.1016/j.ajp.2017.05.004

12. Picard LS, Lindsay S, Strawn JR, Kaneria RM, Patel NC, Keck PE, Jr. Atypical neuroleptic malignant syndrome: diagnostic controversies and considerations. Pharmacotherapy. 2008;28(4):530-5. doi:10.1592/phco.28.4.530

13. Sarkar S, Gupta N. Drug information update. Atypical antipsychotics and neuroleptic malignant syndrome: nuances and pragmatics of the association. BJPsych Bull. 2017;41(4):211-6. doi:10.1192/ pb.bp. 116.053736
14. Koch M, Chandragiri S, Rizvi S, Petrides G, Francis A. Catatonic signs in neuroleptic malignant syndrome. Compr Psychiatry. 2000;41(1):73-5.

15. Fink M TM. Catatonia. A Clinician's Guide to Diagnosis and Treatment. Cambridge: Cambridge University Press; 2003.

16. Northoff G. Catatonia and neuroleptic malignant syndrome: psychopathology and pathophysiology. J Neural Transm (Vienna). 2002;109(12):1453-67. doi:10.1007/s00702-002-0762-z

17. van Rensburg R, Decloedt EH. An Approach to the Pharmacotherapy of Neuroleptic Malignant Syndrome. Psychopharmacol Bull. 2019;49(1):8491.

18. Strawn JR, Keck PE, Jr., Caroff SN. Neuroleptic malignant syndrome. Am J Psychiatry. 2007;164(6):870-6. doi:10.1176/ajp.2007.164.6.870

19. Reulbach U, Dutsch C, Biermann T, Sperling W, Thuerauf N, Kornhuber J, et al. Managing an effective treatment for neuroleptic malignant syndrome. Crit Care. 2007;11(1):4. doi:10.1186/cc5148

20. Trollor JN, Sachdev PS. Electroconvulsive treatment of neuroleptic malignant syndrome: a review and report of cases. Aust N Z J Psychiatry. 1999;33(5):6509. doi:10.1080/j.1440-1614.1999.00630.x

21. Davis JM, Janicak PG, Sakkas P, Gilmore C, Wang Z. Electroconvulsive Therapy in the Treatment of the Neuroleptic Malignant Syndrome. Convuls Ther. 1991;7(2):111-20.

Recibido: 16/11/2019 Aceptado: 19/12/2019 